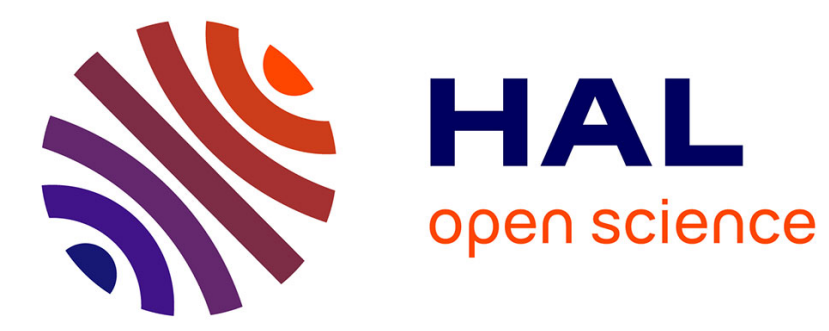

\title{
Laser cooling with a single laser beam and a planar diffractor
}

\author{
Matthieu Vangeleyn, Paul F. Griffin, Erling Riis, Aidan S. Arnold
}

\section{To cite this version:}

Matthieu Vangeleyn, Paul F. Griffin, Erling Riis, Aidan S. Arnold. Laser cooling with a single laser beam and a planar diffractor. Optics Letters, 2010, 35 (20), pp.3. 10.1364/OL.35.003453 . hal01095913

\section{HAL Id: hal-01095913 \\ https://hal.science/hal-01095913}

Submitted on 16 Dec 2014

HAL is a multi-disciplinary open access archive for the deposit and dissemination of scientific research documents, whether they are published or not. The documents may come from teaching and research institutions in France or abroad, or from public or private research centers.
L'archive ouverte pluridisciplinaire HAL, est destinée au dépôt et à la diffusion de documents scientifiques de niveau recherche, publiés ou non, émanant des établissements d'enseignement et de recherche français ou étrangers, des laboratoires publics ou privés. 


\title{
Laser cooling with a single laser beam and a planar diffractor
}

\author{
Matthieu Vangeleyn, Paul F. Griffin, Erling Riis, and Aidan S. Arnold* \\ Department of Physics, Scottish Universities Physics Alliance, University of Strathclyde, Glasgow G4 ONG, UK \\ *Corresponding author: a.arnold@phys.strath.ac.uk \\ Received June 21, 2010; revised August 27, 2010; accepted August 30, 2010; \\ posted September 20, 2010 (Doc. ID 130385); published October 12, 2010 \\ A planar triplet of diffraction gratings is used to transform a single laser beam into a four-beam tetrahedral \\ magneto-optical trap. This "flat" pyramid diffractor geometry is ideal for future microfabrication. We demonstrate \\ the technique by trapping and subsequently sub-Doppler cooling ${ }^{87} \mathrm{Rb}$ atoms to $30 \mu \mathrm{K}$. (C) 2010 Optical Society \\ of America \\ OCIS codes: $\quad 020.1335,140.3320$.
}

A magneto-optical trap (MOT) [1] is the starting point for the vast majority of cold and ultracold atomic physics experiments. Atoms are trapped and cooled to submillikelvin temperatures using light scattering modified by the Zeeman and Doppler effects, respectively. MOTs are typically formed at the center of a spherical quadrupole magnetic field, in the overlap region of six (or less commonly four [2]) appropriately polarized red-detuned laser beams. The original pyramid MOT (PMOT) [3] , utilizing a square-based pyramidal reflector with $90^{\circ}$ apex angle between opposite sides, was devised as a means to turn a single laser beam into the six appropriately polarized beams required for an MOT. The PMOT simplifies optical alignment, saves a large number of optical components, and can also be modified to produce a beam source of cold atoms [4]. The original PMOT has since been used to make a compact gravimeter [5] and a millimeter-scale chip trap [6].

Recently, we demonstrated a new kind of pyramid MOT, based on a four-beam tetrahedral geometry [7] originating from a single beam interacting with a triangular pyramid reflector. This geometry has many advantages over the original design: MOT formation outside the pyramid is possible, which simplifies optical access to the atoms, and the apex region of the pyramid is noncritical (the latter feature is also present in the PMOT design in [8]). The apex and mirror edges in the original PMOT will generate diffraction, and an incorrect apex angle will also generate intensity irregularities in the doubly reflected beams counterpropagating with the input beam. As these irregularities pass directly through the MOT, they can hinder further cooling in optical molasses. Moreover, although sub-Doppler cooling is possible with a small atom number [5] , for larger PMOTs the counterpropagating beam will contain a shadow of the atoms from the input beam, creating an intensity imbalance that will hinder molasses [9]. This problem is obviated in the tetrahedral PMOT.

In this Letter, we have experimentally realized our proposal to extend the tetrahedral PMOT [7] to a "flat" geometry using diffraction gratings. The grating magnetooptical trap (GMOT) has a very similar working principle to the tetrahedral PMOT, and its properties are again largely the result of intensity balance and polarization decomposition [7]. One major difference is due to the fact that gratings spatially compress beams with a corre- sponding intensity increase [Fig. 1(a)]. The relationship between the intensities $I_{i}$ and $I_{1}$ of the vertical incident beam and the first-order diffracted beam, respectively, is determined by the corresponding beam widths $w_{i}$ and $w_{1}$ and the first-order diffraction efficiency $R_{1}$ :

$$
I_{1} / I_{i}=R_{1} w_{i} / w_{1}=R_{1} \sec \alpha,
$$

where the Bragg condition yields the first-order diffraction angle $\alpha=\arcsin (\lambda / d)$ for light with wavelength $\lambda$ normally incident on a grating with groove spacing $d$. Note the relation $\alpha=2 \theta$ allows direct comparison with mirror declination angle $\theta$ in our previous work [7].

The condition for balanced optical molasses from beams with intensities $I_{j}$ and wave vectors $\mathbf{k}_{j}$ is

$$
\sum I_{j} \mathbf{k}_{j}=\mathbf{0}
$$

If we consider the configuration depicted in Fig. 1 , where all but one beam are provided by diffraction from $n$ identical gratings, then Eq. (2) is radially always satisfied, given the symmetry of the problem. Substituting Eq. (1) into Eq. (2) and projecting onto the vertical axis yields the very simple condition for balanced optical molasses:

$$
R_{1}=1 / n
$$

which is completely independent of diffraction angle and, hence, grating period. For three beams, this corresponds to a first-order diffraction efficiency of $R_{1}=1 / 3$.

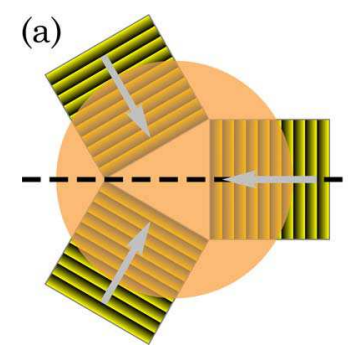

(b)

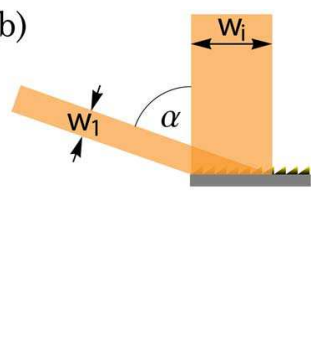

Fig. 1. (Color online) (a) Top view of three gratings and the circular vertical incident beam. The arrows show the blaze direction. (b) Side view of a first-order diffracted beam, illustrating geometric beam compression. Tomographic movies of the beam overlap volume for (a) are shown in Media 1. 
We consider only gratings for which second-order diffraction is absent (i.e., first-order angles $\alpha>30^{\circ}$ ), as they are simpler to model and additionally small $\alpha=2 \theta$ leads to drastically reduced trapping and cooling properties [7].

A critical point in the achievement of a tetrahedral magneto-optical trap is the circular polarization of the first-order diffracted beams. Grating efficiency is usually specified in terms of $S$ and $P$ polarization and can vary dramatically with wavelength and polarization. However, the difference in phase accumulation between $S$ and $P$ components $\phi_{S P}$ also has to be taken into consideration. For our configuration, optimal cooling and trapping is achieved when the handedness (direction of circular polarization relative to beam propagation) of the incident vertical beam is reversed [7] and the total power drops by a factor of 3 (i.e., all beams have equal intensity).

Consider first-order $S$ - and $P$-polarized light components that, after diffraction, have intensities $I_{S}$ and $I_{P}$, respectively, with a relative phase shift of $\phi_{S P}$ (ideally $\pi / 2$ [7]). One can express the reflected beam polarization in electric-field vector components in the plane perpendicular to beam propagation in terms of oppositely handed circular polarizations that trap or antitrap the atoms ( + or - subscripts, respectively) by solving

$$
\left\{\sqrt{I_{S}}, \sqrt{I_{P}} e^{i \phi_{S P}}\right\}=\sqrt{I_{+}} e^{i \phi_{+}}\{1, i\}+\sqrt{I_{-}} e^{i \phi_{-}}\{1,-i\} .
$$

One can then show that the radial trapping constant of the GMOT, relative to a tetrahedral PMOT beam of the same intensity, is reduced by a correction factor:

$$
\zeta_{S P}=\frac{I_{+}-I_{-}}{I_{+}+I_{-}}=2 \sqrt{I_{S} / I_{P}} \sin \left(\phi_{S P}\right) /\left(1+I_{S} / I_{P}\right) .
$$

The effect of relative $S: P$ intensity ratio and phase is shown in Fig. 2 and is surprisingly robust. For an ideal $\pi / 2$ phase shift between $S$ and $P$, even an intensity ratio $I_{S} \sim 0.07 I_{P}$ still yields $\sim 1 / 2$ the trapping strength (black curve in Fig. 2). We measured the efficiency of our gratings for a circularly polarized incident beam to be $R_{1}=45.3 \%$, of which $90 \%$ has the correct circular handedness. If we consider the ensemble made of the grating and the quartz plate of the vacuum chamber (Fig. 3),

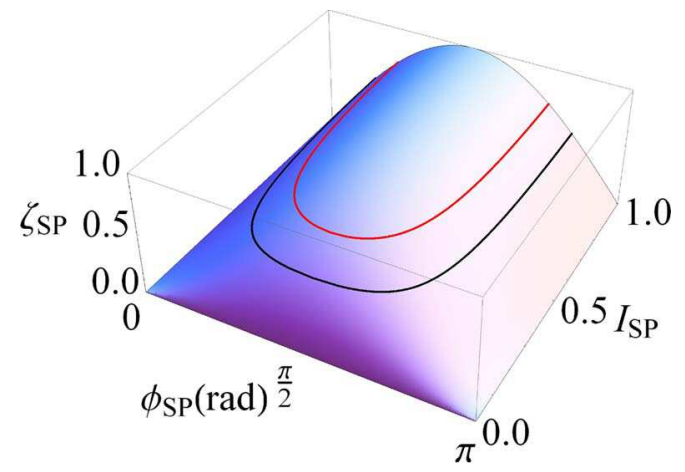

Fig. 2. (Color online) Relative grating MOT radial trapping strength compared to the tetrahedral PMOT [ $\underline{7}], \eta_{S P}$, as a function of the intensity ratio $I_{S P}$ and relative phase $\phi_{S P}$ between the $S$ and $P$ components of the first-order beams at the MOT location. The outer black and inner red curves indicate 50\% and 70\% relative trapping strength, respectively. overall diffraction efficiency drops to a near-optimal $R_{1}=32.2 \%$, with $85 \%$ having the correct handedness, i.e., $\zeta_{S P}=70 \%$ (red curve in Fig. 2).

In the experiment, trapping and repumping light are provided by two independent external-cavity diode lasers [10]. The lasers are overlapped and then spatially filtered by a $30 \mu \mathrm{m}$ pinhole to remove rapid spatial intensity variation before and after diffraction from the gratings, which degrades the trap loading and can prevent subDoppler molasses. The beam is also overexpanded such that the intensity profile is as flat as possible within the $23 \mathrm{~mm}$ diameter apertured laser beam to reduce intensity gradients in the three diffracted beams. A quarter-wave plate changes the polarization to circular just before the vacuum chamber. We used inexpensive Edmund Optics gratings NT43-752. These 1200 grooves/mm gratings deflect a normally incident $780 \mathrm{~nm}$ beam at an angle $\approx 69.4^{\circ}$, close to the ideal tetrahedron PMOT angle $(\arccos (1 / 3) \approx$ $70.5^{\circ}$ ). This yields maximum trapping and cooling [7], albeit with a decreased capture volume using the grating geometry.

The grating triplet is positioned below the glass vacuum cell, the gratings forming a triangle with the blazed direction pointing toward the center [Fig. 1(a)]. The gratings have dimensions $12.7 \mathrm{~mm}$ by $12.7 \mathrm{~mm}$ and, thus, are not completely illuminated by the $23 \mathrm{~mm}$ diameter laser beam. After diffraction, the beams are vertically squeezed to about $w_{1}=2.7 \mathrm{~mm}$, due to compression on the gratings, and the overlap region, where atoms can be trapped, is approximately a flattened rhombohedron [7]. The overlap volume is $\sim 60 \mathrm{~mm}^{3}$ and entirely above the 3 -mm-thick quartz vacuum window (Fig. 3). By deliberately tilting the gratings beyond the flat geometry to increase the beamoverlap region, we found we could collect more atoms, indicating that the atom number is indeed overlap-volume limited. Ideally gratings with a longer period could be used, however, for commercial gratings, the variety in blaze angle (and hence polarization-dependent diffraction efficiency) is limited unless the spatial period is a multiple of 600 grooves $/ \mathrm{mm}$.

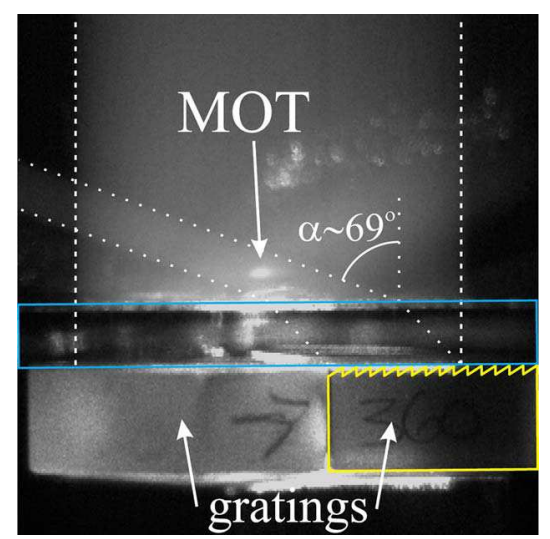

Fig. 3. (Color online) Photograph of the experimental setup from the side. The MOT forms in the overlap of the downward incident beam (dashed white lines) and the three first-order grating beams. The path of the diffracted beam (dotted white lines) from one grating (yellow schematic appearing as a short rectangle in the lower right) refracts through the 3-mm-thick quartz vacuum cell (blue long rectangle that extends across the figure), then propagates at the expected $\alpha \sim 69^{\circ}$. 


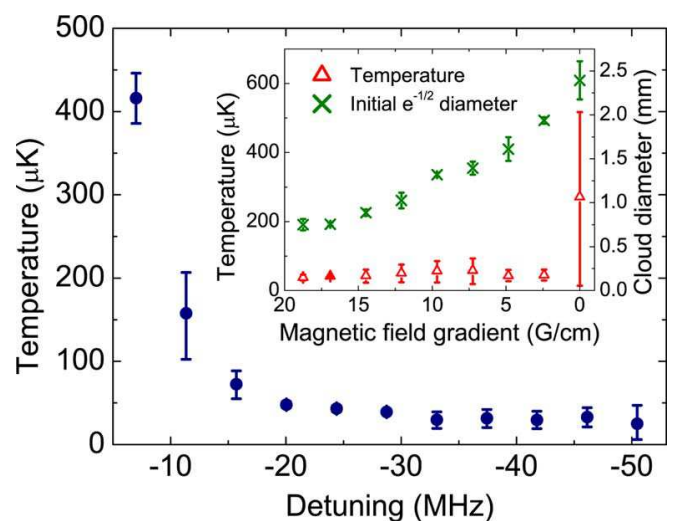

Fig. 4. (Color online) Temperature in the MOT as a function of the $20 \mathrm{~ms}$ extra detuning. For red-detuning jumps larger than $30 \mathrm{MHz}$, the temperature drops to $30 \pm 10 \mu \mathrm{K}$. Inset, temperature and initial $e^{-1 / 2}$ diameter of the atomic cloud if the quadrupole field is reduced to the specified value for the final $10 \mathrm{~ms}$ of $30 \mathrm{MHz}$ sub-Doppler cooling.

With intensities of $1.3 \mathrm{~mW} / \mathrm{cm}^{2}$ in both the vertical and diffracted beams, a magnetic field gradient of $17 \mathrm{G} / \mathrm{cm}$, and $7 \mathrm{MHz}$ red detuning, we trap $10^{5}{ }^{87} \mathrm{Rb}$ atoms in our GMOT (Fig. 3), consistent with the beam-overlap volume reduction from our previous work [7]. For our experimental parameters, the atom number should only enter the volume-squared scaling regime [6] for beam diameters less than $2 \mathrm{~mm}$.

After initial MOT loading, the light frequency is further red detuned for $20 \mathrm{~ms}$ to achieve sub-Doppler $(<140 \mu \mathrm{K})$ MOT temperatures. The cloud temperature is measured using the sizes of background-subtracted fluorescence images after 0 and $10 \mathrm{~ms}$ time of flight. Figure 4 shows the evolution of temperature as a function of extra inMOT detuning, reaching significantly sub-Doppler temperatures of $30 \pm 10 \mu \mathrm{K}$ for red detunings $>30 \mathrm{MHz}$. For a fixed $30 \mathrm{MHz}$ extra red-detuning, we investigated optical molasses formation by reducing the MOT magnetic field gradient to a fixed value in the range $0-17 \mathrm{G} / \mathrm{cm}$ for the last $10 \mathrm{~ms}$ of the $20 \mathrm{~ms}$ in-MOT cooling phase. Although the temperature remains approximately constant, as the final magnetic field gradient reaches zero, the 1/e diameter of the Gaussian cloud prior to time-of-flight imaging reaches a size $(\sim 4 \mathrm{~mm})$ comparable with the beam-overlap volume.

It appears that deeper molasses cooling is largely prevented by spatial intensity variation in our small beamoverlap volume, particularly near the edge of the cloud. Under optimal conditions, we have seen preliminary evidence for optical molasses; however, future experiments would be better performed in an optimized setup-with larger beam overlap and less dramatic diffracted beam compression. Both goals can be achieved using diffraction gratings with a larger groove period. A particularly appealing aspect of the GMOT is that it lends itself to custom microfabricated planar optical elements with arbitrary groove spacing in a stand-alone planar element.

In conclusion, we have demonstrated a pyramid magneto-optical trap with "flat" optics, extending our work on the tetrahedral pyramid MOT [7]. A single beam is split into three new beams by a planar diffractor. This diffractor design is essential for mass-produced microfabrication, as technically challenging and prohibitively time-consuming deep etching is not required. Additionally, MOT formation above the plane of the gratings has clear advantages for detection and further manipulation of the atoms. One can envisage applications in portable MOT-based devices. Moreover, we have demonstrated sub-Doppler temperatures in our grating MOT, and a large-scale GMOT with large atom number has shadow-free beams, which should maintain sub-Doppler optical molasses, suitable for applications requiring Bose-Einstein condensation formation.

We are grateful for stimulating discussions with Joseph Cotter and Ed Hinds. P. F. G. received support from the RSE/Scottish Government Marie Curie Personal Research fellowship program.

\section{References}

1. E. L. Raab, M. Prentiss, A. Cable, S. Chu, and D. E. Pritchard, Phys. Rev. Lett. 59, 2631 (1987).

2. F. Shimizu, K. Shimizu, and H. Takuma, Opt. Lett. 16, 339 (1991).

3. K. I. Lee, J. A. Kim, H. R. Noh, and W. Jhe, Opt. Lett. 21, 1177 (1996).

4. J. J. Arlt, O. Maragò, S. Webster, S. Hopkins, and C. J. Foot, Opt. Commun. 157, 303 (1998).

5. Q. Bodart, S. Merlet, N. Malossi, F. Pereira Dos Santos, P. Bouyer, and A. Landragin, Appl. Phys. Lett. 96, 134101 (2010).

6. S. Pollock, J. P. Cotter, A. Laliotis, and E. A. Hinds, Opt. Express 17, 14109 (2009).

7. M. Vangeleyn, P. F. Griffin, E. Riis, and A. S. Arnold, Opt. Express 17, 13601 (2009).

8. B. M. Xu, X. Chen, J. Wang, and M. S. Zhan, Opt. Commun. 281, 5819 (2008).

9. P. D. Lett, W. D. Phillips, S. L. Rolston, C. E. Tanner, R. N. Watts, and C. I. Westbrook, J. Opt. Soc. Am. B 6, 2084 (1989).

10. A. S. Arnold, J. S. Wilson, and M. G. Boshier, Rev. Sci. Instrum. 69, 1236 (1998). 